\title{
sciendo
}

\author{
ŁUKASZ RAMS ${ }^{1}$, KATARZYNA ŚWITKA ${ }^{2}$, PAULINA KAMIŃSKA ${ }^{3}$, BARTŁOMIEJ KULESZA ${ }^{4}$
}

\section{Brain metastases the challenge of contemporary oncology}

\begin{abstract}
Brain metastases (BM) represent the most common tumours of the central nervous system with ranged between 2.8 and 14.3 per 100.000. Despite advances in the diagnosis and treatment of brain metastases, such as surgery, chemotherapy and radiotherapy only $2.4 \%$ of patients will survive 5 years. BM causes a wide spectrum of neurological symptoms, such as hemiparesis, impaired coordination or walking, aphasia, and seizures. Despite the effective treatment of the primary tumor, in many cases, it does not protect against brain metastases. The main source of BMs in adults is, in descending order, non-small cell lung cancer, followed by breast cancer and melanoma and then renal cancer. Some malignancies particularly tend to produce "late" or "delayed" cerebral metastasis years or even decades after the anti-cancer treatment has been accomplished. There is still a need to develop more effective treatments for cancer and metastases to the brain.
\end{abstract}

Keywords: brain metastases, cancer, blood-brain-barrier, primary tumor.

DOI: $10.2478 /$ pjph-2020-0002

\section{INTRODUCTION}

Brain metastases (BM) represent the most common tumours of the central nervous system. Incidence of new diagnosis of brain metastases is 3 to 10 times higher than of primary malignant brain tumours [1]. Annual incidence in populationbased studies ranged between 2.8 and 14.3 per 100,000 [2-4]. The exact incidence of BMs is unknown and most probably underestimated $[5,6]$, however, based on current estimations, roughly $20-40 \%$ patients with systemic cancer disease will develop a brain metastasis at some point in course of their illness [7]. Almost a half of them will suffer from multiple lesions [8-10]. Large but old autopsy studies on died cancer patients, originating from period when autopsies have been performed routinely, showed a higher incidence that ranged between $9 \%$ and $26 \%[11,12]$. However, since the incidence of BM increases with better control of systemic disease and prolonged time from initial cancer diagnosis, the real prevalence of cerebral metastases might be significantly higher.

The extent of occurrence of BM varies depending on cancer histology and its stage $[13,14]$. At the beginning of past decade roughly 170.000 new diagnoses annually, alone in the USA, have been made [15], which could be translated into approximately 30.000 new diagnoses annually in European country.

\section{Prognosis of patients with BM:}

Despite the fact, that technical advances on the field of diagnosis and treatment of the brain metastases have been made, as well as more efficient treatment regimes of cancer disease utilising different modalities have been established, the prognosis of patients harbouring BMs is poor [16-20]. Taking together, the overall survival in various studies varies between 3 and 20.7 months and 5-year survival is reached by $2.4 \%$ patients $[21,22]$. These numbers depend on diverse factors, among which the negative influence have: poor general condition expressed in lower Karnofsky Performance Status (KPS), progressive status of systemic disease and coexisting extracerebral metastases. Some histologies carry poor prognosis too: median survival for patients with non-small cell lung cancer (NSCLC), even if treated, does not reach 6 months [23]. A 1-year survival rate among these patients is only 10\% [24].

The natural course of untreated BM is a progressive neurological deterioration and eventually, death due to increased intracranial pressure caused by progressively growing lesion(s). The median survival in untreated patients is less than 1 month [25]. Relapse on the primary site after surgical resection followed by adjuvant treatment are very frequent and reach $50 \%$ [24]. Thus, the vast majority of cancer patients (80\%) undergoing a neurosurgical treatment of their BM die due to extracerebral spread or local progression of their systemic disease, not due to its intracranial manifestation $[10,25]$.

Still increasing incidence and poor prognosis regarding the 5-year survival with short time-to-progression is an enormous challenge for the neurooncology of our era. Moreover, BMs, because of wide spectrum of neurological symptoms, cause hemiparesis, coordination or walking disturbance, aphasia, epileptic seizures [26], leading to physical and behavioural

${ }^{1}$ Department of Neurosurgery, AK Altona Hamburg, Germany

${ }^{2}$ Med 1 Clinic, Hamburg, Germany

${ }^{3}$ Department of Clinical Oncology and Chemotherapy, SPSK 4 in Lublinie, Poland

${ }^{4}$ Department of Neurosurgery, Medical University in Lublin, Poland 
disability, and have dramatically negative impact on quality of life of patients carrying this diagnosis [13]. This may negatively affect either the subjective perception of the illness and reduce the effect of anticancer therapy.

\section{Reasons of increased detection of BMs:}

Many reasons could be given why the incidence of BMs is still increasing. Molecular principle of carcinogenesis are damages at the DNA-level accumulated in the human genome over time. After exceeding the point, in which they cannot be restored through the genome self-repair mechanisms, they lead to uncontrolled cellular proliferation. This stage of silent accumulation of genomic mutations may last decades, which explains, why the elderly patients are more likely to develop a cancer with subsequent cerebral spread $[18,28]$.

One of the problems of aggressive anti-cancer treatment is the local evolution within the brain whilst the disease is under control in the extra-cerebral sites [13,28]. With use of novel combined therapy regimes for cancer patients, a good local control and prolonged survival could be achieved. Thus, chemotherapy for systemic cancer may be one of the risks for development of BM [29,30]. Because of their size, hydrophobic molecules of chemotherapeutic agents are not able to cross blood-brain-barrier (BBB) [31], a regulated continuous interface between the peripheral blood circulation and the central nervous system [32]. The BBB is still intact at the early stages of cancer disease and therefore achieving a therapeutic concentration within the brain cannot be maintained, making the brain invasion possible. Once breaching the BBB, the tumour cells may switch into prolonged "hibernation mode" and activate even years or decades after eradicating the systemic cancer.

Improvements in neuroimaging, especially in the field of increasing the imaging quality and accessibility also helped to increase the number of detected BMs [33]. Increased use of neuroimaging devices in routinely controlled neurological asymptomatic cancer patients may be a next reason also. Certain types of cancers have a greater tendency to spread in the central nervous system than others. In the small cell lung cancer (SCLC) the occurrence of brain metastases is very high, neuroimaging is a part of a standard screening after detecting the primary tumour [29]. Modern, more sensitive devices may detect smaller lesions, allowing earlier treatment planning [33]. Cranial magnetic resonance (MRI) replaced computed tomography, mainly due to its higher sensitivity, as the imaging modality of choice for brain tumours almost 40 years ago $[31,34,35]$ but in older MRI-devices lesions smaller than $5 \mathrm{~mm}$ may remain unenhanced. The modern devices provide detection of lesions even $3 \mathrm{~mm}$ of diameter. As many as $20 \%$ of patients with a singular intracranial mass seen in CT-scan have multiple lesions in cranial MRI [35].

\section{Symptoms of brain metastases:}

There is a wide diversity of symptoms that BMS are causing. Symptoms of increased intracranial pressure, such as headaches, nausea and vomiting or progressing impairment of consciousness up to coma may be caused due to obstruction of the circulation pathways of the cerebro-spinal-fluid (CSF) or progressing brain oedema [36]. Tumour causing a mass effect in certain part of the brain, either due its rapid enlargement or local brain swelling, will compress the neural pathways [26]. Localisation in motoric strip or in course of pyramidal tract will result in mono- or hemiparesis on a contralateral side. Involvement of the frontal lobe may cause psychiatric symptoms, lesions in temporal lobe may provoke epileptic seizures. Visual disorders are result of metastasis in occipital lobe. Noteworthy, a pathognomic symptom of BM does not exist and its certain diagnosis, based only on clinical examination without neuroimaging, cannot be made. On the other hand, prevalence of asymptomatic patients is very high: up to $30 \%$ cancer patients harbouring a BM show no neurological disorders at all [37] and the BM would be detected in brain imaging performed for some other reason such as suspicion of cerebral haemorrhage, stroke, after brain trauma or in a routinely conducted follow-up in asymptomatic patient.

\section{The histological source of BM:}

The main source of BMs in adults is, in descending order, non-small cell lung cancer (NSCLC, 35-60\%), followed by breast cancer and melanoma (each 15\%) and then renal cancer (8\% respectively) [18,38,39], accounting overall for from 67 to $88 \%$ of cases. Other histologies, such as thyroid cancer or cancers of gastro-intestinal tract are rare. A challenging issue from the clinical point of view is a patient with malignancy of unknown primary (CUP), in which the source of seeding at the time of diagnosis of BM remains unknown [19]. With the aid of various imaging studies, a primary tumour can be discovered in only up to $40 \%$ patients initially diagnosed with CUP-syndrome [40] and the search process can last months. Typically, the histopathological examination of BMs in CUPsyndrome reveals adenocarcinomas (40-60\%), in other cases they are usually low differentiated cancers.

Two theories explaining the CUP phenomenon with cerebral seeding seem to be probable. According to the first one, the primary cancer regresses spontaneously or has been eliminated by the immune response of the host shortly after microseeding in cerebrum has already occurred. It remains unknown, why the micro-metastasis transforms into macroscopic lesion with latency of months or years, when the primary site has already been eradicated [19], probably it is due to breakdown of the immune system response. The second scenario relies on hypothesis, that for CUP-syndrome is responsible a small and very instable primary tumour of extremely aggressively behaviour, which, because of its high metastatic activity, leads to patient's death before it can transform into detectable macroscopic tumour.

The expression of cerebral metastases differs in different parts of CNS (the prevalence of singular metastases is: frontal and cerebellar $27 \%$, parietal $20 \%$, occipital $14 \%$, temporal $9 \%$ ) [25], but the pathophysiologic mechanism behind this is not clear. It has been considered that region with robust blood flow, as in the grey-white matter junction or cortical brain, are in higher danger of development cerebral metastasis, although prevalence of metastases in other regions of high blood perfusion, such as in brain stem (3\% respectively) [26], is low [28].

\section{Delayed cerebral seeding and histology-related brain seed- ing propensity:}

Some malignancies particularly tend to produce "late" or "delayed" cerebral metastasis years or even decades after the anti-cancer treatment has been accomplished and the tumourfree situation in the extra-cerebral sites has been achieved. This phenomenon characterises melanoma, breast cancer [10], renal and thyroid cancer [41]. Different histological types of 
cancers have different propensity to seed in central nervous system. The highest tendency to metastasize in brain has melanoma, which makes only $1 \%$ of all cancers and is responsible for $15 \%$ BMs. The autopsy reports reveal that $90 \%$ of melanoma patients had simultaneously a cerebral tumour [29]. In comparison, the brain involvement in lung cancer is relatively uncommon (10-20\%), but due to its highest incidence among all cancers it is the main source of BMs. Despite that, lung and breast cancers have a greater tendency to produce multiple seeding in brain [26].

\section{CONCLUSIONS}

Brain metastases are the most common tumors in the CNS. Each CNS symptom in a patient treated for cancer should be an indication for MRI of the brain. Despite various combinations of treatments, such as surgery, chemotherapy and radiotherapy, the prognosis is very poor. Despite the effective treatment of the primary tumor, in many cases, it does not protect against brain metastases. Additionally, some malignancies particularly tend to produce "late" or "delayed" cerebral metastasis years or even decades after the anti-cancer treatment has been accomplished. There is still a need to develop more effective treatments for cancer and metastases to the brain.

\section{REFERENCES}

1. Franchino, F.R. Ruda, Soffietti R. Mechanisms and therapy for cancer metastasis to the rrain. Front Oncol, 201;8:161-70.

2. Prendecka M, Frankowski J, Sobieszek G, et al. Electric Cell Substrate Impedance Sensing (ECIS) as a unique technique in cancer metastasis research. J Pre Clin Clin Res. 2018;12(4):142-4.

3. Counsell CE, Collie DA, Grant R. Incidence of intracranial tumours in the Lothian region of Scotland, 1989-90. J Neurol Neurosurg Psychiatry. 1996;61(2):143-50.

4. Singh R, Stoltzfus KC, Chen H, et al. Epidemiology of synchronous brain metastases. NOA. 2020;2(1):1-10.

5. Fox BD, Cheung VJ, Akash ChJ. Epidemiology of metastatic brain tumors. Neurosurg Clin N Am. 2011;22(1):1-6.

6. Gavrilovic IT, Posner JB. Brain metastases: epidemiology and pathophysiology. J Neurooncol. 2005;75(1):5-14.

7. Kamp MA, Slotty PJ, Cornelius JF, et al. The impact of cerebral metastases growth pattern on neurosurgical treatment. Neurosurg Rev. 2018;41(1):7786.

8. Rostami R, Mittal S, Rostami P, et al. Brain metastasis in breast cancer: a comprehensive literature review. J Neurooncol. 2016;127(3):407-14.

9. Ferguson SD, Wagner KM, Prabhu SS, et al. Neurosurgical management of brain metastases. Clin Exp Metastasis. 2017;34(6-7):377-89.

10. Schackert G, Steinmetz A, Meier U, et al. Surgical management of single and multiple brain metastases: results of a retrospective study. Onkologie. 2001;24(3): 246-525

11. Rutkowski P, Kiprian D, Dudzisz-Śledź M, et al. Management of brain metastases in melanoma. Oncol Clin Pract. 2019;15(1):51-61.

12. Posner JB, Chernik NL. Intracranial metastases from systemic cancer. Adv Neurol. 1978;19:79-92.

13. Martinive P, Van Houtte P. The challenge of brain metastases from nonsmall cell lung cancer is not only an economical issue. Ann Palliat Med. 2019;8(2):203-6.

14. Mazurek M, Litak J, Kamieniak P, et al. Metformin as potential therapy for high-grade glioma. Cancers. 2020;12:210.

15. Claus EB. Neurosurgical management of metastases in the central nervous system. Nat Rev Clin Oncol. 2012;9(2):79-86.

16. Daphu I, Sundstrøm T, Horn S, et al. In vivo animal models for studying brain metastasis: value and limitations. Clin Exp Metastasis. 2013;30(5):695-710.

17. Zhang X, Zhang W, Cao WD, et al. A review of current management of brain metastases. Ann Surg Oncol. 2012;19(3):1043-50.
18. Schödel P, Schebesch KM, Brawanski A, et al. Surgical resection of brain metastases-impact on neurological outcome. Int J Mol Sci. 2013;14(5):8708-18.

19. Preusser M, Capper D, Ilhan-Mutlu A et al. Brain metastases: pathobiology and emerging targeted therapies. Acta Neuropathol. 2012;123(2):205-22.

20. Kulesza B, Mazurek M, Nogalski A, et al. Factors with the strongest prognostic value associated with in-hospital mortality rate among patients operated for acute subdural and epidural hematoma. Eur J Trauma Emerg Surg. (2020). https://doi.org/10.1007/s00068-020-01460-8

21. Fabi A, Felici A, Metro G, et al. Brain metastases from solid tumors: disease outcome according to type of treatment and therapeutic resources of the treating center. J Exp Clin Cancer Res. 2011;30(1):10.

22. Hall WA, Djalilian HR, Nussbaum ES, et al. Long-term survival with metastatic cancer to the brain. Med Oncol. 2000;17(4):279-86.

23. Song, Z. Zhang Y. Gefitinib and erlotinib for non-small cell lung cancer patients who fail to respond to radiotherapy for brain metastases. J Clin Neurosci. 2014;21(4):591-5.

24. Al-Zabin, M, Ullrich WO, Brawanski A, et al. Recurrent brain metastases from lung cancer: the impact of reoperation. Acta Neurochirurgica. 2010;152(11):1887-92.

25. Korinth MC, Delonge C, Hütter BO. Prognostic factors for patients with microsurgically resected brain metastases. Onkologie. 2002;25(5):420-5.

26. Biswas G, Bhagwat R, Khurana R, et al. Brain metastasis - evidence based management. J Can Res Ther. 2006;2(1):5-13.

27. Schebesch KM, Hoehne J, Hohenberger C, et al. Fluorescein sodium-guided resection of cerebral metastases - experience with the first 30 patients. Acta Neurochirurgica. 2015;157(6):899-904.

28. Hoshide, R. Jandial R. The role of the neural niche in brain metastasis. Clin Exp Metastasis. 2017;34(6-7):369-76.

29. Alexandru DD, Bota A, Linskey MW. Epidemiology of central nervous system metastases. Prog Neurol Surg. 2012;25:13-29.

30. Patchell RA, Tibbs PA, Walsh JW, et al. A randomized trial of surgery in the treatment of single metastases to the brain. NEJM. 1990;322(8):494500 .

31. Klotz R, Thomas A, Teng T, et al. Circulating tumor cells exhibit metastatic tropism and reveal brain metastasis drivers. Cancer Discov. 2020;10(1):86-103.

32. Arshad, F, Wang L, Sy Ch, et al. Blood-brain barrier integrity and breast cancer metastasis to the brain. Patholog Res Int. 2010;2011:920509.

33. Ranasinghe MG, Sheehan JM. Surgical management of brain metastases. Neurosurg Focus. 2007;22(3):E2

34. Pope WB. Brain metastases: neuroimaging. Handb Clin Neurol. 2018;149:89-112.

35. Bindal RK, Sawaya R, Leavens ME, et al. Surgical treatment of multiple brain metastases. JNS. 1993;79(2):210-6.

36. Sunderland GJ, Jenkinson MD, Zakaria R. Surgical management of posterior fossa metastases. J Neurooncol. 2016;130(3):535-42.

37. Perrakis A, Juratli TA, Hohenberger W, et al. Surgery for metastases, anatomical and ethical limits. Special aspect: oligometastases. Chirurg. 2016;87(3):208-15.

38. Mut M. Surgical treatment of brain metastasis: a review. Clin Neurol Neurosurg. 2011;114(1):1-8.

39. Al-Shamy G, Sawaya R. Management of brain metastases: the indispensable role of surgery. J Neurooncol. 2009;92(3):275-82.

40. Zaun, G, Schuler M, Herrmann K, et al. CUP Syndrome-Metastatic Malignancy with Unknown Primary Tumor. Dtsch Arztebl Int. 2018;115(10):157-62.

41. Gomes-Lima CJ, Wu D, Raoet SN, et al. Brain Metastases From Differentiated Thyroid Carcinoma: Prevalence, Current Therapies, and Outcomes. J Endocr Soc. 2019;3(2):359-71.

\section{Corresponding author}

Dr Bartłomiej Kulesza

Department of Neurosurgery,

Medical University in Lublin

e-mail: kuleszabartek88@gmail.com 